\title{
Role of Catecholamine Pathways in Action of Orexin B Induced Open Field Activity by Nucleus Accumbens in Wistar Rats
}

\author{
Rashmi Kaup Shiva', Ganaraja Bolumbu²*, Santosh Mayannavar ${ }^{3}$, Dharnappa Poojari ${ }^{3}$
}

\section{Rashmi Kaup Shiva ${ }^{1}$, Ganaraja Bolambu ${ }^{2 *}$, Santosh Mayannavar ${ }^{3}$}

'Department of Physiology, Centre for Basic Sciences, Kasturba Medical College (A unit of Manipal Academy of Higher Education (MAHE), Manipal), Mangalore, Karnataka, INDIA.

${ }^{2}$ Department of Physiology, Karwar Institute of Medical Sciences, Karwar, Karnataka, INDIA.

${ }^{3}$ Department of Oral and Maxillofacial Surgery, Manipal College of Dental Sciences, Mangaluru, Karnataka, INDIA.

Correspondence

Dr. Ganaraja B

PhD. Additional Professor, Department of Physiology, Centre for Basic Sciences, Kasturba Medical College, (A Unit of Maniapl Academy of higher Education MAHE, Manipal), Mangalore, Karnataka, INDIA.

Phone no : 9449642150

E-mail: ganaraja.b@manipal.edu

History

- Submission Date: 05-02-2018.

- Review completed: 06-03-2018

- Accepted Date: 19-04-2018

DOI : 10.5530/pj.2018.4.103

Article Available online

http://www.phcogj.com/v10/i4

\section{Copyright}

(C) 2018 Phcog.Net. This is an openaccess article distributed under the terms of the Creative Commons Attribution 4.0 International license.

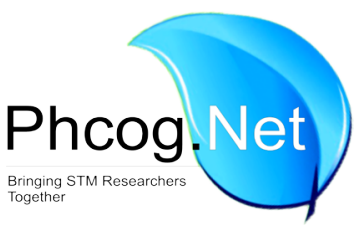

ABSTRACT

Aims: To elucidate whether exploratory behaviour changes following Orexin B infusion in NAc is mediated through catecholamines in male Wistar rats. Methods: Inbred male Wistar rats $(n=24)$ were divided into three groups. Control, Treated 1 (Orexin B) and Treated 2 (Orexin B antagonist) groups. Using stereotaxic method, guide cannula was set in place bilaterally to reach Nucleus Accumbens. Orexin B and its antagonist, TCS-OX2-29 were infused in separate groups of overnight fasted rats. Following open field activity, catecholamines (Dopamine, Adrenaline, and Noradrenaline) were estimated in brain tissue homogenate by ELISA. Data were expressed as mean \pm SEM (ANOVA; Student-Newman Keuls test,).p $<0.05$ were considered as statistically significant. Results: Orexin B infusion significantly increased noradrenaline levels in Nucleus accumbens when compared to controls. TCS-OX2-29 significantly decreased noradrenaline in Nucleus accumbens during open field exploration activity. Adrenaline was not altered significantly during open field activity. Conclusion: These results suggest that Orexin B, which plays a role in the regulation of motor and exploratory behaviour when infused in NAc mediates these actions through noradrenergic neurotransmission in Nucleus Accumbens.

Key words: Adrenaline, Dopamine, Nucleus Accumbens, Noradrenaline, Orexin B, TCSOX2-29.

\section{INTRODUCTION}

Motivated behaviours are basically maintained by Wakefulness and vigilance levels. Orexins - a pair of neuropeptides that are essential for maintaining wakefulness - were also involved in the regulation of motivated behaviors such as feeding and drinking and implicated in emotional aspects. ${ }^{1}$ Orexin $\mathrm{A}$ and Orexin B are primarily identified in the lateral hypothalamus, the classical feeding center. ${ }^{2}$ Their receptors, OX1 and OX2 have been expressed in several places in the brain. ${ }^{3}$ Moderate numbers of Orexin fibres were located in medial Nucleus Accumbens (NAc). ${ }^{4}$

NAc is implicated in motivated behaviours like feeding and drinking via dopamine receptors. ${ }^{5,6}$ It was reported that the Orexin system regulates central motor control. ${ }^{7}$ Infusion of Orexin A into paraventricular nucleus of hypothalamus augmented the impulsive physical exploratory activity in rats, whereas $\mathrm{OX}_{1} \mathrm{R}$ antagonist SB334867 significantly attenuated such effects. ${ }^{8}$ Orexin A infusion into the rostral lateral part of hypothalamus, $\mathrm{NAc}^{9}$ and locus coeruleus increased locomotor activity in rats. ${ }^{10}$ Orexin $\mathrm{A}$ induced firing in local coeruleus, indicated potential involvement of Orexin in maintaining arousal. We earlier demonstrated that Orexin B infusion into Nucleus Accumbens increased exploratory behaviour, but not anxiety like behaviour. ${ }^{11}$ This was proved by inhibition of this activity by Orexin B antagonist (TCS-OX2-29), which decreased exploratory behaviour significantly, whereas anxiety behaviour was unaffected.

Dopamine action in the Nucleus Accumbens has been implicated in motivated behaviours. ${ }^{12}$ Further dopamine antagonist suppressed increased locomotor activity by Orexins in Rodents..$^{13}$ Role of glutamate in Orexin A induced motivated behaviour is also well established. ${ }^{14}$ Role of serotonergic system in the Orexin infused locomotor behaviour is also demonstrated. ${ }^{15}$ Studies indicate that noradrenaline transmission increases locomotor activity. ${ }^{16}$ Yet data on Orexin induced catecholaminergic pathway in exploratory behaviour is unclear.

Hence in the present study was designed to investigate if the alteration in exploratory behaviour by Orexin $\mathrm{B}$ and its antagonist is due to adrenergic and/or noradrenergic activity in Nucleus Accumbens. To explore the possible mechanism involved in Orexin B mediated exploratory behaviour standard biochemical analysis of adrenaline and noradrenaline in Nucleus Accumbens were conducted immediately after the experimental session.

\section{MATERIALS AND METHODS}

A total of 48 male albino Wistar rats of 3-4 months old (200-275gms) were housed separately in poly-

Cite this article: Rashmi KS, Ganaraja B, Mayannavar S, Poojary D. Role of Catecholamine Pathways in Action of Orexin B Induced Open Field Activity by Nucleus Accumbens in Wistar Rats. Pharmacog J. 2018;10(4):628-32. 
propylene cages $(29 \mathrm{cms} \times 22 \mathrm{cms} \times 14 \mathrm{cms})$ with bedding of paddy husk, under normal day-night cycle in temperature controlled room during the experimental period. Food pellets (procured from Pranav Agro Industries Ltd, Maharashtra, India) and potable tap water were available to animals $a d$. Lib. (except in the groups as mentioned in the experimental requirement). Animals were divided into Group I (Control) Group II (Orexin B) and Group III (Oexin B antagonist) with 6 animals each.

Animals were done according to the notifications by Government of India. (Government of India gazette notification, Dec 15, 1998.). Prior to the initiation of the study Institutional Animal Ethical Committee (I.A.E.C) (KMC/57/2009-2010) consent was obtained.

\section{METHODOLOGY}

\section{Surgical procedure and cannulation}

A combination of Ketamine $(50 \mathrm{mg} / \mathrm{ml})$ and Xylazine $(20 \mathrm{mg} / \mathrm{ml})$ were used to anesthetize the rats. Calculated volume of anesthetic agents was drawn from the vials to make a solution containing the equivalent of $70 \mathrm{mg} / \mathrm{kg}$ bwtt of Ketamine and $10 \mathrm{mg} / \mathrm{kg}$ body weight of Xylazine and injected by i.p. route. Stereotaxic surgical procedure and implantation of cannula was done according to our previously published work. ${ }^{17,18,19}$

\section{Micro infusion procedure}

Orexin B (30nanomol/ $\mu$ l) (Catalog no. 06262 Sigma Aldrich, St Louis, USA) was administered bilaterally into the specific nuclei in separate groups of rats. ${ }^{20}$ TCS-OX2-29 (Catalog.No.3370; Tocris Bioscience, UK), a Orexin B antagonist, was dissolved in $0.9 \%$ saline and injected at a dose of 10 micrograms/ $\mu$ in separate groups of rats. ${ }^{17}$ Sham controls with the surgical procedure and injected with $0.9 \%$ saline. The infusion Orexin $\mathrm{B}$ and TCS-OX2-29 was performed according to the methodology published in the earlier work of current laboratory. ${ }^{6}$

Rats were made to fast for a day prior to drug infusion. Four trials of Orexin B infusion was carried out. $72 \mathrm{~h}$ intervened between consecutive treatment days. Open field exploration test was carried out as described earlier. $^{11}$

\section{Estimation of neurotransmitters \\ Dissection of discrete regions of the brain}

Sample collection was done next day of the fourth trial of Orexin B/ antagonist infusion into the Nucleus Accumbens and BLA. Rat brain was removed soon after sacrifice of the rats by decapitation. The dorsal portion of the cranium was peeled off by bone cutter and by means of blunt forceps brain was placed into ice-cold glass plate excluding the olfactory bulbs. Coronal sections of $1 \mathrm{~mm}$ thickness were made in rat brain slicer and were mounted on glass slide. Nucleus Accumbens and Basolateral amygdala were quickly dissected out on a chilled petridish placed on crushed ice.

\section{Tissue preparation}

The freshly prepared brain sample on thin aluminium foil, was freezed and stored immediately at $-70^{\circ} \mathrm{C}$ or deeper until subsequent preparation. At the day of homogenization the wet weight of the sample (NAc and BLA) was estimated and transferred it into ice cooled solution containing $0.1 \mathrm{~N} \mathrm{Hcl}$ and $1 \mathrm{mmol}$ EDTA ( $1 \mathrm{ml} / 50 \mathrm{mg}$ wet weight brain tissue). Centrifugation of the sample was done at $15,000 \mathrm{~g}$ for $15 \mathrm{~min}$ at $4^{\circ} \mathrm{C}$ and the supernatant were used for ELISA analysis.

Noradrenaline and adrenaline were estimated by Tricat ELISA kit (IBL, Germany catalogue number.RE-59395). The optical density (OD) was measured using Bio Tek $\mathrm{EL}_{\mathrm{x}}-800$ automated ELISA micro plate reader.

\section{Statistical analysis}

Data is represented as mean \pm SEM. One way ANOVA followed by Student-Newman Keuls test was used. (SPSS version.16.0.) $p<0.05$ was considered significant.

\section{RESULTS}

Effect of micro infusion of orexin B and TCS-OX2-29 into Nucleus Accumbens on adrenaline and noradrenaline in Openfield exploration test

The infusion of orexin B into Nucleus Accumbens made significantly more $(p<0.001)$ central square entries compared to controls (Figure 1$)$. There was also a significant increase $(p<0.001)$ in time spent in central squares compared to controls (Figure 2). Nucleus Accumbens infused group didn't show any alteration in the number of rearings, $(p>0.05)$ grooming $(p>0.05)$ and fecal excreta $(p>0.05)$ (Figure 3$)$. The infusion of Orexin B antagonist (TCS-OX2-29) into Nucleus Accumbens made significantly fewer $(p<0.01)$ central squares entries compared to controls (Figure 1). There was also a significant decrease $(p<0.001)$ in time spent in central squares compared to controls (Figure 2). Orexin antagonist infusion in Nucleus Accumbens also didn't show any alteration in the number of rearing, $(p>0.05)$ grooming $(p>0.05)$ and fecal excreta ( $>0.05)$ (Figure 3)

\section{Effect of Orexin B and its antagonist on adrenaline and noradrenaline}

Orexin B infusion into NAc significantly increased Accumbal noradrenaline levels $(0.66 \pm 0.05)$ with respect to controls $(0.48 \pm 0.06)$ during Open field exploration test $(\mathrm{p}<0.05)$. Orexin B antagonist infusion decreased the noradrenaline levels in accumbal region $(0.24 \pm 0.03)$ compared to controls $(0.48 \pm 0.06)(\mathrm{p}<0.01)$ in open field exploration test. No significant differences in adrenaline and noradrenaline levels were observed between any of the groups.

\begin{tabular}{|c|c|c|c|c|}
\hline \multirow[b]{2}{*}{ Neurotransmitters } & \multicolumn{3}{|c|}{ Nucleus Accumbens } & \multirow[b]{2}{*}{ F value significance } \\
\hline & Control & $\begin{array}{c}\text { Treated } \\
\text { (orexin B) }\end{array}$ & $\begin{array}{c}\text { Treated } \\
\text { (TCS-0x2-29) }\end{array}$ & \\
\hline Adrenaline(ng/ml) & $0.23 \pm 0.02$ & $0.26 \pm 0.01$ & $0.27 \pm 0.02$ & \\
\hline Noradrenaline(ng/ml) & $0.48 \pm 0.06$ & $0.66 \pm 0.05^{*}$ & $0.24 \pm 0.03^{s s}$ & $\begin{array}{l}\mathrm{P}<0.001 \\
\mathrm{~F}=19.03\end{array}$ \\
\hline
\end{tabular}

[Data are expressed as mean \pm SEM. $(n=6)$ (One way ANOVA; Student - Newman- Keuls. multiple comparison test $\left.]^{*} \mathrm{p}<0.05, \$ \$ p<0.01\right]$ 


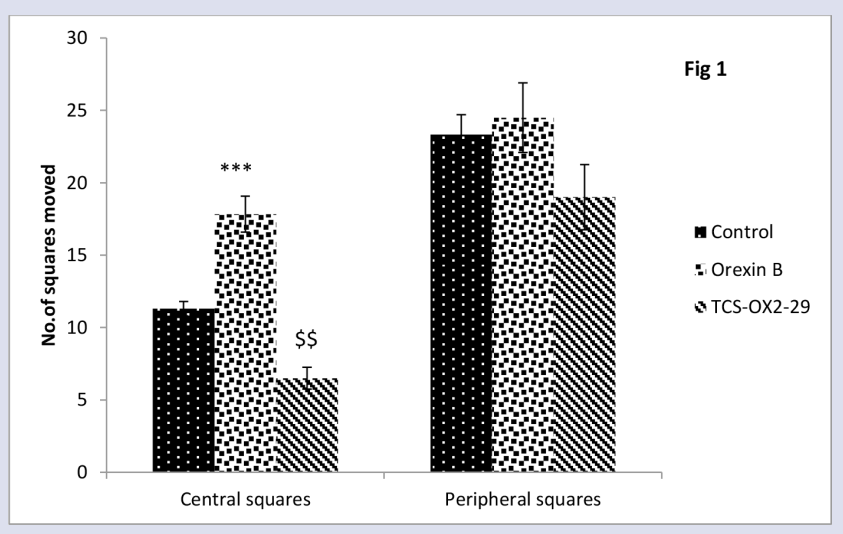

Figure 1: Effect of central infusion (nucleus accumbens) of orexin $B$ and orexin B antagonist TCS-OX2-29 on the number of central and peripheral squares moved. Repeated measures ANOVA, Student Newman-Keuls. multiple comparison test, ${ }^{* * *}=p<0.001$.

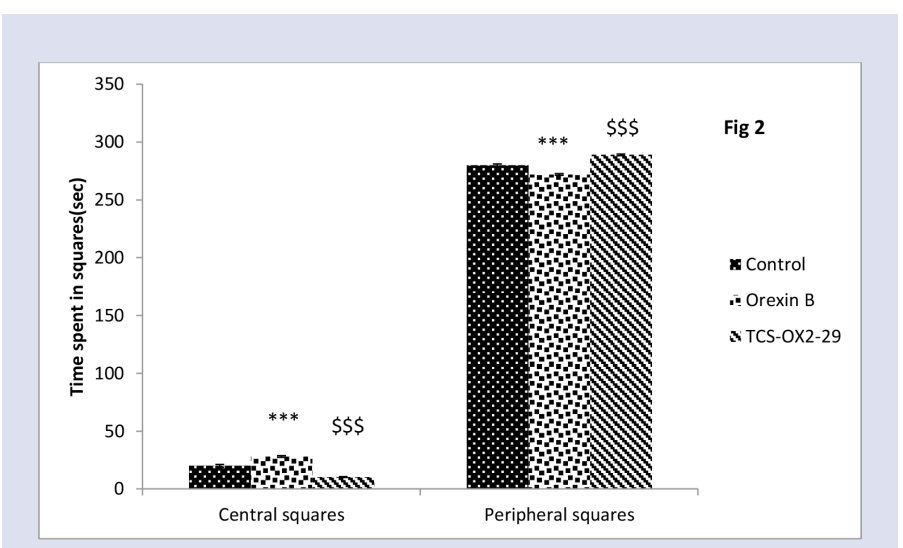

Figure 2: Effect of Orexin B, its antagonist infused to Nucleus Accumbens on the

the time spent in central and peripheral squares. Repeated measures ANOVA, Student-Newman- Keuls. multiple comparison test, ${ }^{* * *}=p<0.001$.

\section{Summary of open field in Orexin B and antagonist infused rats in Nucleus Accumbens.}

Note :Graph is reproduced from results presented in our previous article published from our lab11

\section{DISCUSSION}

The nature of relationship between neuropeptides Orexin and arousal remains poorly understood. ${ }^{19}$ The earlier reports from our laboratory proved that Orexin B infusion into Nucleus Accumbens stimulates exploratory behaviour. ${ }^{11}$ In our previous study, infusion of Orexin B into NAc induced significantly more central square entries in injected rats when compared to controls. ${ }^{11}$ There was also a significant increase in time spent in central squares compared to controls. ${ }^{11}$ Findings of the present study reveal the significant increase noradrenaline levels in Orexin B infused rats after open field exploration test (Table 1). However, Adrenaline was not significantly altered with Orexin B infusion. Thorpe et al reported earlier that Orexin A infusion into NAc Shell increases locomotor activity ${ }^{21}$

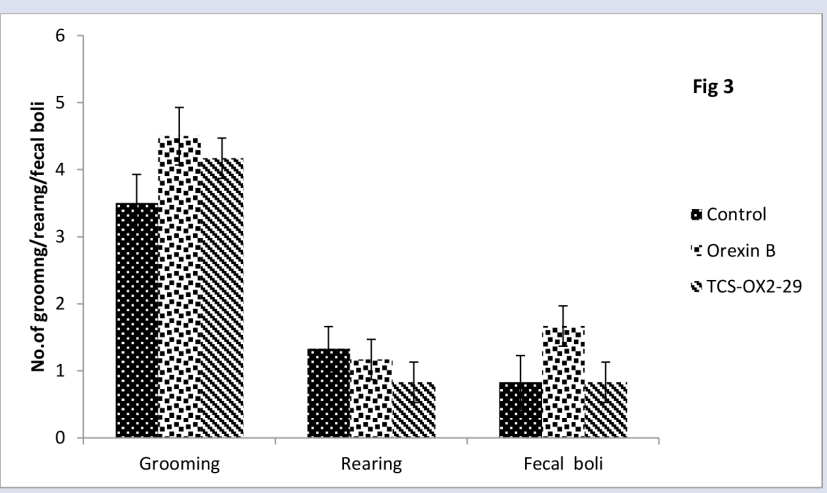

Figure 3: Effect of Orexin B, its antagonist infused to Nucleus Accumbens on the number of grooming, rearing and fecal boli (3) in the open field exploration test in Wistar rats. Data as mean \pm SEM. $(n=6)$ (Repeated measures ANOVA, Student -Newman- Keuls. multiple comparison test)

*** $\mathrm{p}<0.001$, control vs. Orexin B.

$\$ \$ p<0.01, \$ \$ \$ 0.001$ control vs. TCS-OX2-29

Unequivocal evidence for the orexinergic output mediation through the noradrenergic neurons was observed by infusion of orexin antagonist TCS-OX2-29 into the NAc, resulted in significant decrease in noradrenaline levels in the present study. In TCS-OX2-29 infused rats open field exploration activity was reduced. (Table 1). However, Adrenaline was not significantly altered with Orexin B antagonist infusion. Earlier reports from the our laboratory revealed that micro infusion of Orexin $B$ antagonist (TCS-OX2-29) into NAc induced significantly fewer central squares entries and significantly less time spent when compared to controls. ${ }^{11}$ Infusion in Orexin B antagonist didn't alter no of grooming, rearing and fecal boli excreted significantly which is an indicative of anxiety like behaviour. ${ }^{11}$

Findings of the present study reveal the significant increase noradrenaline levels in Orexin B infused rats after open field exploration test (Table 1). Similarly adrnaline was decreased with TCS-OX2-29 infusion. However, Adrenaline was not significantly altered with Orexin B and its antagonist infusion. Li and Vanden Pol reported that Orexin of hypothalamic origin increases arousal which is also a action of noradrenaline. ${ }^{22}$ It was documented that Orexin A potentiated the contraversive pivoting. This was due to the activation of the dopamine receptors in the shell of NAc. Orexin A antagonist SB334867 did not affect the pivoting. ${ }^{23}$

Since discovery of Orexins in 1998, evidence for its role in arousal has been enormous. Studies reinforced OX's role in promoting arousal and wakefulness. ${ }^{24}$ Studies also demonstrate the role of OX in the regulation of a variety of emotional behaviour. ${ }^{25}$ Noradrenergic transmission is directly associated with exploratory behaviour and novelty. ${ }^{26}$ The observed exploratory behaviour in the present study could be due to noradrenergic activity in Nucleus Accumbens. It has long been reported that Noradrenaline in Nucleus Accumbens stimulates locomotor behaviour in rats. ${ }^{27}$ It has also been reported that Orexins activate the neurons containing noradrenaline. ${ }^{28}$ Another study reported that both Orexin peptides release noradrenaline from cerebrocortical slices. ${ }^{29}$

Noradrenaline is one of the important neurotransmitter involved in arousal and sleep wake regulation. Norepinephrine through the ascending arousal system impacts sleep wake cycle. ${ }^{30}$ It is also reported that norepinephrine and locus coeruleus system integrated to regulate sleep and waking. ${ }^{31}$ Orexins also play an important physiological role in maintaining sleep- wake cycle. ${ }^{32}$ In the present study Orexin B infusion 
in to Nucleus Accumbens increases locomotor activity through noradrenergic system. This gives further evidence for the relationship between orexinergic and noradrenergic system. We unequivocally prove that Orexin-noradrenergic system in Nucleus Accumbens stimulate locomotor activity and motivated behaviour.

\section{ACKNOWLEDGEMENT}

This study was supported and funded by Department of Biotechnology, Ministry of Science and Technology, New Delhi; Government of India (Grant.No.BT/PR13878/MED/30/303/2010). We also acknowledge Manipal Academy of Higher Education, Manipal for their support.

\section{ABBREVIATIONS}

BLA: Basolateral amygdala; EDTA: Ethylene Diamine Tetra acetate; ELISA: Enzyme linked immune sorbent assay; I.A.E.C: Institutional Animal Ethics committee; i.p: intra peritonial; NAc: Nucleus Accumbens; OX1 and OX2: Orexin 1 \& Orexin 2; $\mathbf{O X}_{1} \mathbf{R}$ : Orexin one receptor; OX: Orexin; OD: Optical density; SEM: Standard error mean.

\section{CONFLCIT OF INTEREST}

The authors declare no conflict of interest.

\section{REFERENCES}

1. Sakurai T. The role of orexin in motivated behaviours. Nature Reviews Neuroscience. 2014;15(11):719.

2. De Lecea LK, Peyron TS, Gao C, Foye X, Danielson PE, Fukuhara PE, et al. The hypocretins: hypothalamus-specific peptides with neuroexcitatory activity. Proc Natl Acad Sci U S A. 1998;95(1):322-7.

3. Peyron C, Tighe DK, Van Den Pol AN, de Lecea L, Heller HC, Sutcliffe JG, et al. Neurons containing hypocretin (orexin) project to multiple neuronal systems. J Neurosci. 1998;18(23):9996-10015.

4. Rashmi K, Mayannavar S, Deshpande K, Ganaraja B. Effect of orexin B and OX2-R antagonist micro-infusion into the nucleus accumbens on consummatory behaviour in wistar rats. European Journal of Biotechnology and Bioscience. 2014;2(4):30-6

5. Qi K, Wei C, Li Y, Sui N. Orexin receptors within the nucleus accumbens shell mediate the stress but not drug priming-induced reinstatement of morphine conditioned place preference. Frontiers in behavioral neuroscience. 2013;7.

6. Mayannavar S, Rashmi K, Rao YD, Yadav S, Ganaraja B. Effect of Orexin A antagonist (SB-334867) infusion into the nucleus accumbens on consummatory behavior and alcohol preference in Wistar rats. Indian journal of pharmacology. 2016;48(1):53.

7. Hu B, Yang N, Qiao Q-C, Hu Z-A, Zhang J. Roles of the orexin system in central motor control. Neuroscience and Biobehavioral Reviews. 2015;49:43-54.

8. Kiwaki K, Kotz CM, Wang C, Lanningham-Foster L, Levine JA. Orexin A (hypocretin 1) injected into hypothalamic paraventricular nucleus and spontaneous physical activity in rats. American Journal of Physiology - Endocrinology and Metabolism. 2004;286(4):E551-E9

9. Thorpe AJ, Kotz CM. Orexin A in the nucleus accumbens stimulates feeding and locomotor activity. Brain Res. 2005;1050(1-2):156-62.

10. Zink AN, Perez-Leighton CE, Kotz CM. The orexin neuropeptide system: physical activity and hypothalamic function throughout the aging process. Frontiers in systems neuroscience. 2014;8.

11. Rashmi K, Bhat BM, Maynnavar S, Ganaraja B. Does Orexin B Infused into Nucleus Accumbens Affect Open Field Activity In Wistar Rats? Research Journal of Pharmaceutical, Biological and Chemical Sciences. 2016;7(5):2166-70.

12. Salamone JD, Correa M, Farrar A, Mingote SM. Effort-related functions of nucleus accumbens dopamine and associated forebrain circuits. Psychopharmacology. 2007;191(3):461-82

13. Nakamura T, Uramura K, Nambu T, Yada T, Goto K, Yanagisawa M, et al. Orexin- induced hyperlocomotion and stereotypy are mediated by the dopaminergic system. Brain research. 2000;873(1):181-7.

14. Borgland SL, Chang S-J, Bowers MS, Thompson JL, Vittoz N, Floresco SB, et al. Orexin A/hypocretin-1 selectively promotes motivation for positive reinforcers. Journal of Neuroscience. 2009;29(36):11215-25.

15. Matsuzaki I, Sakurai T, Kunii K, Nakamura T, Yanagisawa M, Goto K. Involvement of the serotonergic system in orexin-induced behavioral alterations in rats. Regulatory peptides. 2002;104(1-3):119-23.

16. Darracq L, Blanc G, Glowinski J, Tassin JP. Importance of the noradrenalinedopamine coupling in the locomotor activating effects of D-amphetamine. Journal of neuroscience. 1998;18(7):2729-39.

17. Mayannavar S, Rashmi K, Rao YD, Yadav S, Ganaraja B. Effect of Orexin-A infusion in to the Nucleus Accumbens on consummatory behaviour and alcohol preference in male Wistar rats. 2014.

18. Rashmi K, Mayannavar S, Deshpande K, Ganaraja B. Involvement of Neuropeptide Orexin B in Basolateral Amygdala Mediated Consummatory Behaviour in Male Wistar Albino Rats. 2015.

19. Santhosh Mayannavar RKS, Keerthi Deshpande, Sheila R Pai, Ganaraja B. Preparation of Durable Cannula for Intracranial micro-infusion of NeuroactiveSubstances in Small Animals. International Journal of Innovative Research in Science, Engineering and Technology. 2013;2(11):6032-8.

20. Edwards CM, Sunter A, Murphy D, Ghatei KG, Bloom SR. The effect of the orexins on food intake: comparison with neuropeptide $Y$, melanin-concentrating hormone and galanin. J Endocrinol. 1999;160(3):R7-12.

21. Thorpe AJ, Levine AS, Kotz CM. Centrally administered orexin A increases motivation for sweet pellets in rats. Psychopharmacology. 2005;182(1):75-83.

22. Li Y, Van Den Pol AN. Direct and indirect inhibition by catecholamines of hypocretin/orexin neurons. Journal of Neuroscience. 2005;25(1):173-83.

23. Kotani A, Ikeda $H$, Koshikawa N, Cools AR. Role of orexin receptors in the nucleus accumbens in dopamine-dependent turning behaviour of rats. Neuropharmacology. 2008;54(3):613-9.

24. Chemelli RM, Willie JT, Sinton CM, Elmquist JK, Scammell T, Lee C, et al. Narcolepsy in orexin knockout mice: molecular genetics of sleep regulation. Cell. 1999;98(4):437-51.

25. Kayaba $Y$, Nakamura A, Kasuya $Y$, Ohuchi T, Yanagisawa M, Komuro I, et al. Attenuated defense response and low basal blood pressure in orexin knockout mice. American Journal of Physiology-Regulatory, Integrative and Comparative Physiology. 2003;285(3):R581-R93.

26. Alttoa A. Neurochemical regulation of rat exploratory behaviour: focus on dopaminergic and noradrenergic neurotransmission. 2008.

27. Herman ZS. The effects of noradrenaline on rat's behaviour. Psychopharmacology. 1970;16(5):369-74

28. Yamanaka A, Tsujino N. Willie, Junko Hara, Natsuko Tsujino,Michihiro Mieda, 6 MakotoTominaga, Ken-ichiYagami, Fumihiro Sugiyama, KatsutoshiGoto, Masashi Yanagisawa, and Takeshi Sakurai. Hypothalamic Orexin Neurons Regulate ArousalAccording to Energy Balance in Mice. Neuron. 2003;38:701-13.

29. Hirota K, Kushikata T, Kudo M, Kudo T, Lambert DG, Matsuki A. Orexin A and $B$ evoke noradrenaline release from rat cerebrocortical slices. British journal of pharmacology. 2001;134(7):1461-6.

30. Mitchell HA, Weinshenker D. Good night and good luck: norepinephrine in sleep pharmacology. Biochemical pharmacology. 2010;79(6):801-9.

31. Aston-Jones G, Gonzalez M, Doran S. Role of the locus coeruleus-norepinephrine system in arousal and circadian regulation of the sleep-wake cycle. Brain norepinephrine: Neurobiology and therapeutics. 2007:157-95.

32. Piper DC, Upton N, Smith MI, Hunter AJ. The novel brain neuropeptide, orexin-A, modulates the sleep-wake cycle of rats. European Journal of Neuroscience. 2000;12(2):726-30. 
GRAPHICAL ABSTRACT

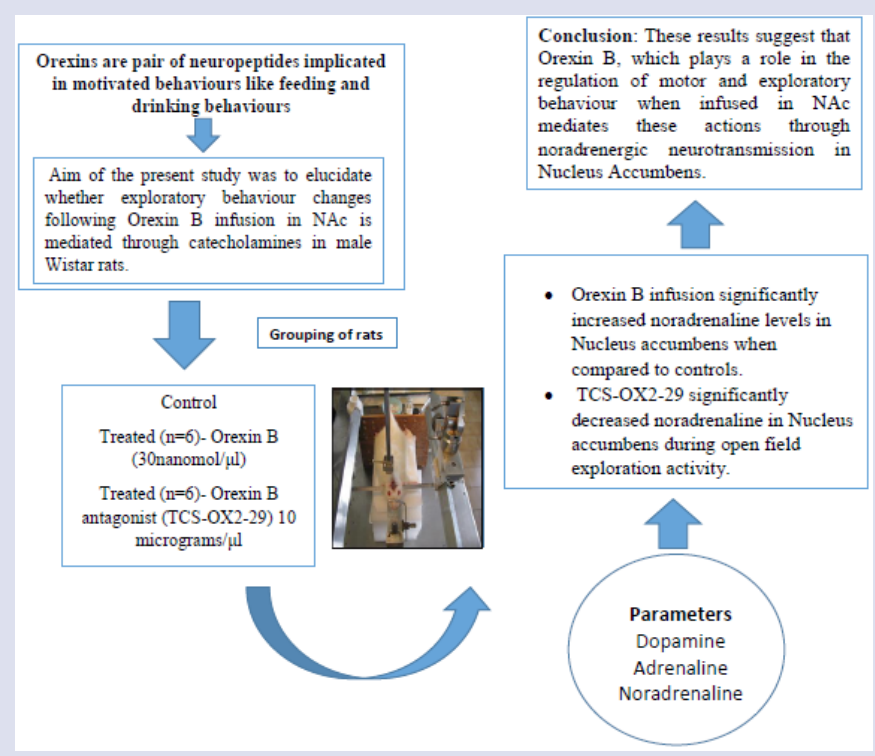

Dr. Ganaraja B, PhD: Additional Professor Published 59 full length papers in peer reviewed journals. Presented more than 35 conference papers. Attended national and international conferences.Carried out extramurally funded project. Guided student research projects. Guided PhD students.

\section{SUMMARY}

- The nature of relationship between neuropeptides Orexin and arousal remains poorly understood. Earlier reports from our laboratory proved that Orexin B infusion into Nucleus Accumbens stimulated exploratory behaviour. In our previous study, infusion of Orexin B into NAc induced significantly more central square entries in injected rats when compared to controls. Findings of the present study reveal the significant increase noradrenaline levels in Orexin B infused rats after open field exploration test. Orexin antagonist TCS-OX2-29 into the NAc, resulted in significant decrease in noradrenaline levels in the present study, revealing that Norepinephrine is involved in the control of open field activity modulated by nucleus accumbens.

\section{ABOUT AUTHORS}

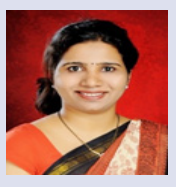

Dr Rashmi KS: Is currently working as an Assistant Professor, in the department of Physiology, KMC, Mangalore, Manipal Academy of Higher Education, Karnataka, India. She has completed her PhD in Neurophysiology in 2016 under Manipal University. She is a member of Japan Neuroscience Society. Her honors include travel grant of 500 euros by Federation of European Neuroscience Society (FENS) to attend neuroscience meeting in Hungary, travel grant of 2500 USD by International Brain Research Organization (IBRO) to attend Japan neuroscience meeting and for a short stay to get trained in research in Japan, She has also received a grant of Rs. 50,000 from Science and Engineering Research Board (SERB) to attend South Asian Association of Physiology conference in Nepal and received the best paper presentation. Her research interests are addiction, herbal medicine and medical education. She has published more than 20 papers in peer reviewed indexed national and international journals. 